\title{
Preventive bundle approach decreases the incidence of ventilator- associated pneumonia in newborn infants
}

\author{
Alejandro Pinilla-González $\mathbb{1}^{1} \cdot$ Álvaro Solaz-García ${ }^{1}$ - Anna Parra-Llorca $•$ Inmaculada Lara-Cantón $\mathbb{D}^{1}{ }^{1}$ \\ Ana Gimeno ${ }^{1,2} \cdot$ Isabel Izquierdo ${ }^{1,2} \cdot$ Máximo Vento $\mathbb{D}^{1,2} \cdot$ María Cernada $\mathbb{D}^{1,2}$
}

Received: 7 November 2020 / Revised: 19 April 2021 / Accepted: 29 April 2021 / Published online: 25 May 2021

(c) The Author(s), under exclusive licence to Springer Nature America, Inc. 2021

\begin{abstract}
Objective We hypothesized that the implementation of evidence-based interventions shaping a bundle approach could significantly reduce the incidence of ventilator-associated pneumonia (VAP) in the neonatal intensive care unit.

Study design We conducted a prospective observational cohort study including neonates undergoing mechanical ventilation $>48 \mathrm{~h}$. VAP rate and endotracheal intubation ratio were compared before (pre-period) and after (post-period) applying VAP prevention bundle strategies.

Result One hundred seventy-four neonates were included in pre-period (30 months) and 106 in post-period (17 months). Demographic characteristics were comparable and device use ratios were similar. Twenty-eight VAP episodes were diagnosed, 25 in the first period and 3 after the implementation of prevention bundle. This represents a reduction in the incidence rate from 11.79 to 1.93 episodes/1000 ventilator days $(p<0.01)$.

Conclusion The implementation of an educational evidence-based program using a bundle approach to prevent VAP has shown a statistically significant reduction in its incidence density.
\end{abstract}

\section{Introduction}

Ventilator-associated pneumonia (VAP), as defined by the Center of Disease Control (CDC) (Atlanta, USA), is an episode of pneumonia diagnosed in patients undergoing mechanical ventilation (MV) for $\geq 48 \mathrm{~h}$. It represents the second most common healthcare-associated infection among infants, with rates that range from 2.7 to 10.9 episodes per 1000 days of MV in the neonatal intensive care units (NICU) in high-income countries [1,2]

Although there are no specific diagnostic criteria for VAP in the newborn period, the CDC criteria for infants $<1$ year old requires clinical, analytical, and radiographic

Máximo Vento

maximo.vento@uv.es

$\checkmark$ María Cernada

mariacernada@gmail.com

1 Neonatal Research Group, Health Research Institute La Fe, Valencia, Spain

2 Division of Neonatology, University and Polytechnic Hospital La Fe, Valencia, Spain findings to assess its diagnosis [3]. Nevertheless, microbiological isolation is not mandatory and the CDC admits the diagnosis of "clinically defined pneumonia" based only on clinical and radiological criteria. Moreover, the most suitable way to obtain samples is not well defined. Thus, while some authors advocate for more accessible techniques such as tracheal aspirates, others prefer to access the lower respiratory tract using non-bronchoscopic bronchoalveolar lavage [4].

VAP is usually caused by the aspiration of contaminated fluids into the lung. Endogenous microorganisms reach the lung from colonized naso/oropharynx, gastric fluid pool, and tracheal secretions. In addition, pathogens can also reach the lung from exogenous sources such as hands of caregivers, ventilator circuits, and endotracheal tube (ETT) biofilms. Polymicrobial etiology is found in $25-40 \%$ of VAP and Staphylococcus aureus, Pseudomonas aeruginosa, and Klebsiella pneumoniae are the most frequently isolated agents $[1,4]$.

The risk factors for developing VAP vary according to different studies; however, most authors agree that both the duration of MV and low birth weight are among the principal contributing factors [5]. The role of sedation, tracheal 
suction, enteral feeding, transfusions, or antibiotics yet remains unclear [2, 6].

VAP is associated with increased morbidity, mortality, and prolonged length of hospital stay [7] representing a significant economic burden for the health system and the families [8].

To our knowledge, only few studies have evaluated the impact of strategies to reduce VAP incidence in infants [1, 2, 9-11]. Nevertheless, they are not focused exclusively on neonates and the diagnostic criteria employed include "clinical pneumonia" and microbiological diagnosis in tracheal aspirates, both constituting a source of bias.

We hypothesized that the implementation of comprehensive evidence-based interventions shaping a bundle approach could significantly reduce the incidence of microbiologically diagnosed VAP.

\section{Methods}

\section{Study design}

A prospective observational cohort study was conducted in the NICU of University and Polytechnic La Fe Hospital, Valencia (Spain) from January 2016 to December 2019. This is a tertiary level unit with 4800-5000 births and 300-350 admissions in NICU per year. The study was approved by the Ethics Committee and parents of all patients signed the informed consent.
The 48 months of the study was divided in two subperiods with a wash-out period of 1 month in between. The pre-period consisted in an observational period of 30 months from January 2016 to June 2018 and the postperiod, in which the bundle was applied, lasted 17 months from July 2018 to December 2019. In between a wash-out period of 1 month was devoted to training.

In the pre prevention period, the staff proceeded with the routine care protocol.

Optimization of non-invasive respiratory support, early enteral feeding with human fresh milk when available and donor human milk or formula depending on gestational age when mothers could not provide own milk, and criteria for intubation and diagnosis of infection were the same in both periods. We adopted in both periods a restricted policy of antibiotic use, a targeted antifungal prophylaxis and a bundle approach to prevent CLABSI. We do not use probiotics in our unit.

Educational intervention (during July 2018) was based in an updated analysis of the scientific literature adapted to our NICU. All health care providers received information and instructions about evidence-based recommendations and were trained in the bundle approach to prevent VAP.

Once the period of implementation of VAP prevention strategies was completed the therapeutic protocol for patients undergoing MV included the VAP prevention bundle. The fulfillment of the requirements included in the bundle was supervised by the nurses in charge who completed an ad hoc check list. All data were collected from the
Fig. 1 Flow chart shows the intervention across time. Preperiod consisted in an observational period from January 2016 to June 2018. Post-period, after the bundle application, from July 2018 to December 2019. Educational intervention was performed in a wash-out period of 1 month in July 2018.
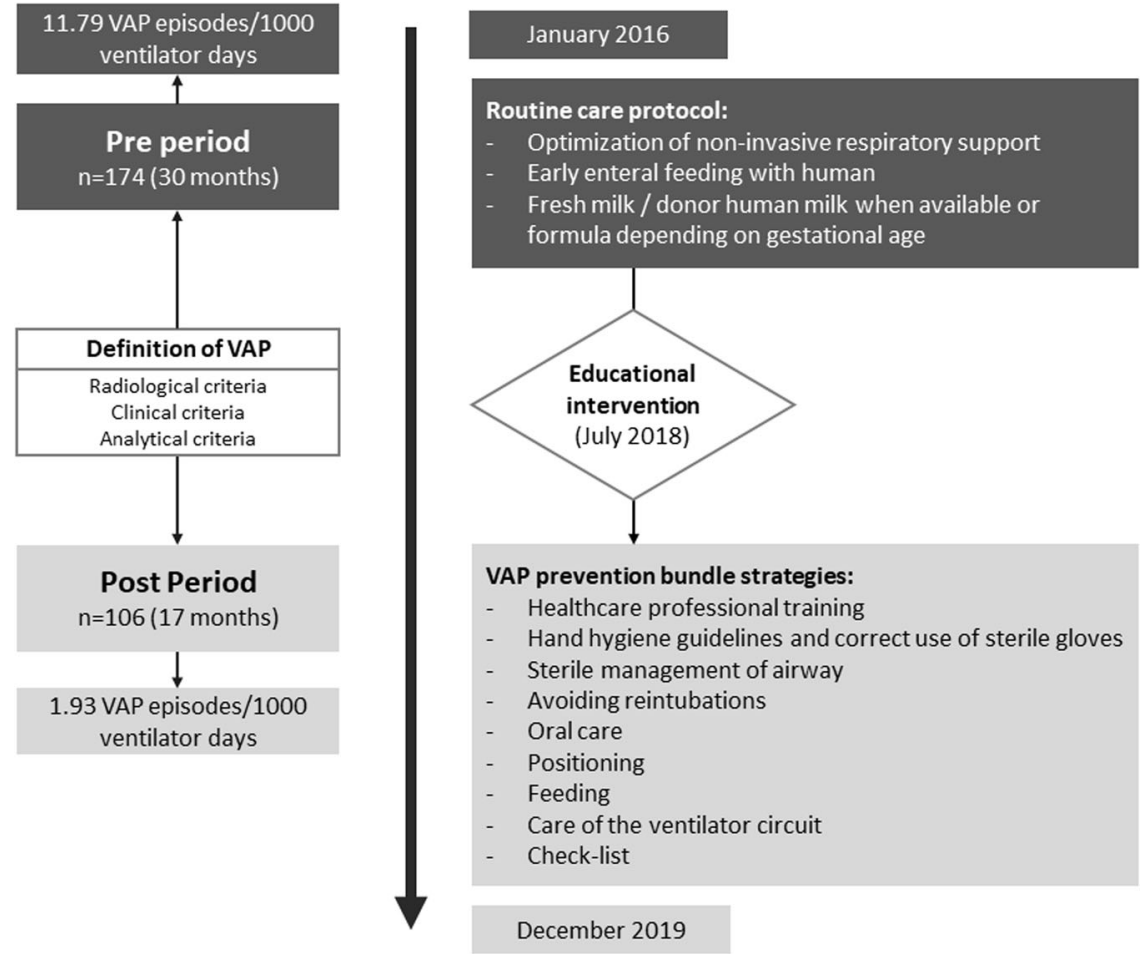
database and electronic clinical records of NICU. Flow chart in Fig. 1 shows the intervention across time.

\section{Patients}

Newborn were eligible and recruited if they were admitted in the NICU and received $\geq 48 \mathrm{~h}$ of $\mathrm{MV}$.

\section{Definition of VAP}

Diagnosis of a VAP episode was based on CDC criteria [3] and our previous experience $[4,5,12]$ and required a combination of radiological, clinical, and analytical criteria.

- Radiological criteria: new or progressive infiltrates, cavitation, consolidation, or pneumatoceles.

- Clinical criteria: worsening of gas exchange; and three of the following criteria: temperature instability; white blood cell count $<4000 / \mathrm{mm}^{3}$ or $>15,000 / \mathrm{mm}^{3}$; newonset in purulent sputum or increase of respiratory secretion; apnea or tachypnea; wheezing or rales or rhonchi; cough; heart rate $<100 \mathrm{bpm}$ or $>170 \mathrm{bpm}$.

- Analytical criteria: positive culture of alveolar lavage using a blind-protected catheter $\left(>10^{3} \mathrm{CFU} / \mathrm{ml}\right)$.

VAP was suspected in patients intubated more than $48 \mathrm{~h}$ with radiological and clinical signs. In these neonates we performed complete blood count, C-reactive protein, blood culture, and a bronchoalveolar lavage using a blindprotected catheter (BAL) through the ETT as previously described [5] to confirm the diagnosis. Empiric antibiotics were started following the NICU protocol. If VAP was confirmed 7-10 days of antibiotics were completed. On the other hand, if VAP was not confirmed and clinical progress was adequate; attending doctors could consider discontinuing treatment [6].

\section{VAP prevention bundle strategies}

(1) Healthcare professional training.

A specific training for each preventive strategy was made to all health-care providers [10].

(2) Adherence to hand hygiene guidelines and correct use of sterile gloves prior to the management of ventilation equipment/supplies.

To avoid oral cavity colonization during tracheal suction and device handling hand hygiene was performed following the WHO recommendations [13] (Fig. 1). Besides, surgical handwashing was made before invasive procedures. The use of sterile gloves was limited to handling ventilator devices [9-11].

(3) Sterile management of airway.

Total barrier prevention strategies were employed during endotracheal intubation and surfactant administration (sterile gloves, gown, cap, and mask). Disinfection of ETT connections was made with $2 \%$ chlorhexidine in $70 \%$ alcohol wipes with every handling.

The suction procedure was made according to the NICU protocol. Procedure should be conducted by two nurses to guarantee aseptic technique, only when ETT was obstructed and only when indispensable and with a double suction system: one for the oral cavity and another for the airway both connected to a close-suction system. ETT instillation of saline solution or distilled water was avoided [10, 11].

(4) Avoiding reintubations.

The nurse-to-patient ratio was kept 1:2 and the need for MV was daily re-evaluated [9-11].

\section{(5) Oral care.}

Cleaning of gums, tongue, and lips with a sterile swab or gauze coated in distilled water was performed every $3-4 \mathrm{~h}$, and before gastric tube insertion, intubation, and ETT repositioning [9-11] to prevent and reduce microbial inoculation.

\section{(6) Positioning.}

To decrease gastric microaspirations, postural changes alternating lateral position with decubitus were performed. Whenever gastroesophageal reflux was observed the head of the bed (HOB) was elevated $15^{\circ}-30^{\circ}[9-11]$.

\section{(7) Feeding.}

Tube feeding in $60-120 \mathrm{~min}$ was preferred over continuous feeding or bolus. Continuous feeding has been related to changes in gastric $\mathrm{pH}$ which could promote gramnegative bacterial colonization, while feeding bolus has been linked with microaspirations [10, 14].

(8) Care of the ventilator circuit.

The breathing circuit of the ventilators was changed only when visibly soiled or malfunctioning [12]. Two percent chlorhexidine in $70 \%$ alcohol was used to clean ETT connections and the self-inflating bags [9-11]. 
Fig. 2 Ventilator-associated pneumonia (VAP rates) (episodes per 1000 ventilator days) and endotracheal tube (ETT) use ratio in \%. The vertical line separates the preand post-bundle approach application.

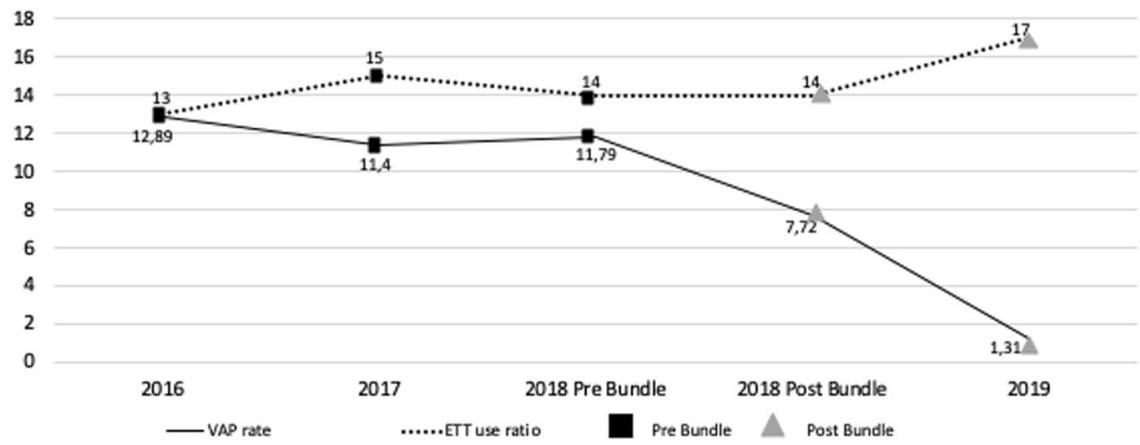

\section{Statistical analysis}

Kolmogorov-Smirnov test was performed to evaluate the normal distribution of our variables. To check if the two cohorts were comparable $\mathrm{Chi}^{2}$ test and Mann-Whitney $U$ test were used according to the nature of the variables. Subsequently, both the pooled incidence density VAP rate (number of VAP cases $\div$ ventilator days $\times 1000$ ) and the device use ratio (number of intubated patients $\div$ number of admitted patients) were calculated for each period. All rates were provided weekly. A day of MV was considered when a patient was intubated for $>12 \mathrm{~h}$. The incidence rate and odds ratio were calculated with $95 \%$ confidence interval (CI). Finally, Mantel-Haenszel $\mathrm{Chi}^{2}$ was used to compare VAP rates in each period. Kaplan-Meier method was used to describe actuarial freedom from VAP over MV period. The log-rank test was used to compare VAP incidence among the period pre and post-intervention. Absolute risk reduction (ARR) and number needed to treat (NNT) were calculated. We considered statistical significance $p<0.05$. Statistical analysis was performed with SPSS Statistics 20.0.

\section{Results}

\section{Analysis of prevention bundle care effect}

A significant reduction in the pooled incidence density of VAP was observed in the overall post-period (1.93 episodes/1000 ventilator days) compared to pre-period (11.79 episodes $/ 1000$ ventilator days). $\mathrm{OR}=5.0766(95 \% \mathrm{CI}$ 1.5328-16.8139). Mantel-Haenszel $\mathrm{Chi}^{2}=8.7686(p=$ 0.00306). Prevented fraction exposure of 0.80 (95\% CI $0.347-0.940$ ), prevented fraction in population of 0.30 (95\% CI 0.39-0.21), incidence ratio 0.1970 (95\% CI 0.0595-0.6524), ARR 0.115, and NNT 8.7.

Microorganisms isolated in first period were E.coli (30\%), K.pneumoniae (25\%), S.aureus (15\%), P.aeruginosa

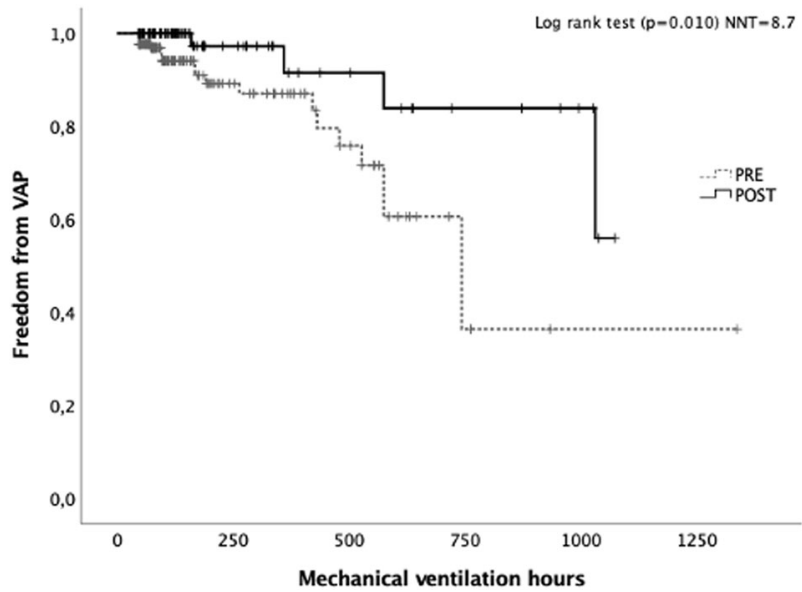

Fig. 3 Freedom from VAP in pre-intervention period $(n=175$ patients) compared to the post-intervention period $(n=106$ patients). MV hours indicates time expressed in hours in which patients were exposed to MV. NNT number needed to treat.

(10\%), and E.gergoviae, E.aerogenes, S.maltophilia, S.marcescens (5\% each one). In second period microorganisms isolated were P.aeruginosa (75\%) and A. baumanii (25\%).

This decrease of VAP rates was not associated with a lower ETT use ratio since it remained similar or even higher, as represented in Fig. 2. The probability of remaining free from VAP during MV was significantly higher in the post-intervention group (log-rank test, $p=$ 0.016), as represented in Fig. 3.

No significant differences in mortality, bronchopulmonary dysplasia, MV days, or length of stay were observed between infants included in both periods (Table 1). A reduction in mortality $(21.3 \%$ pre-period, $13.2 \%$ post-period) was identified but did not reach statistical significance $(p 0.110, p$ 0.833). A trend toward reduction in the use of CVC was observed (pre-period $100 \%$, post-period $95.3 \% ; p<0,004$ ), but total days of CVC were similar. 
Table 1 Demographics of patients pre-intervention and post-intervention to reduce ventilator-associated pneumonia in the NICU.

\begin{tabular}{|c|c|c|c|}
\hline & $\begin{array}{l}\text { Pre-intervention (Jun } \\
\text { 2016-May 2018) } \\
N=174\end{array}$ & $\begin{array}{l}\text { Post-intervention (Jul } \\
\text { 2018-Dec 2019) } \\
N=106\end{array}$ & $p$ value \\
\hline Gestational age(weeks), Median (IQR) & $30(26,38)$ & $30(25,37)$ & 0.970 \\
\hline Birth weight $(\mathrm{kg})$, mean $\pm \mathrm{SD}$ & $1.82 \pm 1.1$ & $1.80 \pm 1.1$ & 0.829 \\
\hline Male, $n(\%)$ & $113(64.9 \%)$ & $106(60.4 \%)$ & 0.442 \\
\hline Cesarean-section, $n(\%)$ & $71(40.8 \%)$ & $45(42.5 \%)$ & 0.786 \\
\hline Apgar (1 min), Median (IQR) & $7(4,9)$ & $6(4,8)$ & 0.479 \\
\hline Apgar (5 min), Median (IQR) & $9(7,10)$ & $8(7,9)$ & 0.428 \\
\hline Death during hospitalization, $n(\%)$ & $37(21.3 \%)$ & $14(13.2 \%)$ & 0.110 \\
\hline Bronchopulmonary dysplasia, $n(\%)$ & $23(13.1 \%)$ & $17(16.7 \%)$ & 0.479 \\
\hline Any surfactant dose, $n(\%)$ & $72(41.4 \%)$ & $46(43.4 \%)$ & 0.740 \\
\hline Re admission in NICU, $n(\%)$ & $9(5.2 \%)$ & $3(2.8 \%)$ & 0.348 \\
\hline Days of NIMV, Median (IQR) & $3(0,29)$ & $5(0,33)$ & 0.633 \\
\hline Days of IMV, Median (IQR) & $5(3,12)$ & $5(3,11)$ & 0.833 \\
\hline Days on oxygen, Median (IQR) & $4(0,20)$ & $4(0,31)$ & 0.638 \\
\hline Number of reintubations, $n(\%)$ & $27(15.5 \%)$ & $25(23.6 \%)$ & 0.113 \\
\hline Number of accidental extubations, $n(\%)$ & $10(5.8 \%)$ & $9(8.5 \%)$ & 0.464 \\
\hline Number CVC, $n(\%)$ & $174(100 \%)$ & $101(95.3 \%)$ & 0.004 \\
\hline CVC days, Median (IQR) & $11(6,19)$ & $11(6,21)$ & 0.748 \\
\hline Days in NICU, Median (IQR) & $14(7,52)$ & $17(8,55)$ & 0.268 \\
\hline Antibiotics, $n(\%)$ & $146(83.9 \%)$ & $94(88.7 \%)$ & 0.268 \\
\hline Absence enteral nutrition, $n(\%)$ & $76(43.7 \%)$ & $43(40.6 \%)$ & $<0.001$ \\
\hline
\end{tabular}

To check if the two cohorts were comparable $\mathrm{Chi}^{2}$ test and Mann-Whitney $U$ test were used according to the nature of the variables.

$C V C$ central venous catheter, NIMV no invasive mechanic ventilation, IMV invasive mechanic ventilation, Days of NIMV $>12 \mathrm{~h}$ periods, Days of IMV >12 h periods.

\section{Discussion}

An effective implementation of VAP strategies has led to a significant reduction in the incidence of VAP in our unit. In the 2016-2018 period, before the bundle approach program was established, our NICU experienced an increase in the incidence from 10.9 to $11.79 \mathrm{VAP} / 1000$ ventilator days although the use of MV remained similar. The pooled density rate published in 2016 by the International Nosocomial Infection Control Consortium in a multicentric epidemiologic surveillance in 50 countries was 9.2/1000 ventilator days [15]. Nevertheless, the device use ratio in this report $(0.26)$ was higher than ours (0.15).

Several studies carried out in the adult and pediatric populations have shown that bundle programs are useful in preventing VAP episodes in patients admitted to intensive care. Indeed, bundle programs have been able to achieve a reduction of $20 \%$ of the VAP rates [16-18]. Studies in newborns have already shown that bundle strategies can be successfully applied in the NICU $[1,2,9-11]$. However, in some of these studies, patients with the diagnosis of "clinical pneumonia" or without microbiological confirmation $[2,11,12,17]$ were included. Both these circumstances could bias the diagnosis and evolving rates of VAP in non-blinded studies. Moreover, tracheal aspirate frequently reflects colonization instead of infection and leads to VAP over-diagnose thus overestimating the impact of the preventive interventions [19]. Our diagnostic method [5] includes a combination of clinical and radiological criteria but also the microbiological isolation. Furthermore, the non-invasive blind-protected BAL allowed for reaching the lower airway tract avoiding contamination from the colonized upper airway. Our approach avoids contamination and facilitates a reliable microbiological diagnosis and the reduction of bias [20]

Focusing on preventive period measures, hand hygiene has been included in all of bundle VAP care described since it has been shown to be the most important measure to reduce nosocomial infections [9-11, 16, 17].

Oral care especially in pediatric patients has been controversial. While some authors recommend the routine use of antiseptic solutions and tooth brushing [11, 16, 17], others prefer normal saline and suction of oropharyngeal secretions [18]. However, according to specific studies in the neonatal population that agree with the use of normal 
saline solution because of the lack of teeth in newborns [9-11], we included this strategy in our bundle care.

Elevation of head of bed to reduce gastric microaspiration is also comprised in most of the studies [16-18]. However, a disparity of grades of elevation in neonatal articles from $10^{\circ}$ to $45^{\circ}$ is noted $[9,11]$. In our study, the highest elevation was of $12^{\circ}$ because is the maximum elevation that the design of our incubators allow. This is in accordance with Weber et al. [10].

Bolus feeding has been related to gastric distension which could facilitate the microaspiration of gastric content to the pharynx and reach the lower respiratory airways. Continuous feeding, however, induces changes of gastric $\mathrm{pH}$ which can promote gram-negative microorganism proliferation [17, 21-24]. We decided to standardize feeding rhythm using 60-120 min gastroclisis.

Finally, in our study the airway management included: sterile management of the airway, avoiding reintubation and unnecessary changes of breathing circuit, and optimizing the management of the MV. We proposed cleaning respiratory device connections with $2 \%$ chlorhexidine in $70 \%$ alcoholic solution [10] after each handling in agreement with our protocol for central lines management since a greater bactericidal effect of chlorhexidine in alcohol has been described $[10,11]$. Every one of these procedures was made as strictly indicated by unit protocol.

Some studies have applied all the bundle at once $[9,16,17,25,26]$, while others have implemented it progressively to determine the impact of each intervention [3]. We chose to comprehensively implement the bundle in our study.

The adherence rate to our VAP bundle care was high according to check list fulfillment. Hence, a physician collected all VAP suspicions, use of central lines, number of intubated patients, and confirmed VAP episodes daily.

Our bundle VAP prevention strategy has decreased VAP rate from 12.89 episodes/1000 ventilator days in 2016 to 1.31 in 2019. This result means a NNT close to 9, rendering our bundle highly effective from a clinical perspective. A similar study has been published by Weber et al. but the final impact has not been reported [10]. Other studies have shown a further VAP rate decrease with similar strategies but it could be related to a higher pre-bundle incidence of VAP [27].

We did not find a significant reduction in relevant clinical outcomes although there was a tendency toward a reduction in mortality. Herewith, we acknowledge limitations in this regard. Hence, the sample calculation did not include mortality as an additional outcome because given the low incidence of VAP in our unit it would have excessively prolonged the duration of our study. In this regard, we plan to launch a multicenter trial to answer questions that yet remain unknown. However, the application of our bundle in settings with a higher VAP incidence may possibly have a positive influence on outcomes such as mortality, length of stay, and/or days on MV.

\section{Conclusion}

We conclude that the implementation of an educational evidence-based program using a bundle approach to prevent VAP has led to a statistically significant reduction in the incidence density and a tendency toward clinical improvement.

Acknowledgements We would like to thank all the doctors and nurses of the staff for their effort and enthusiasm implementing the bundle in our NICU.

Author contributions AP-G, MC, IL-C, and AP-L designed the data collection instruments, recruited the patients, collected data, and reviewed and revised the manuscript. AP-G, AS-G, and MC carried out the analyses, and reviewed and revised the manuscript. MC, AG, II, and MV conceptualized and designed the study, coordinated and supervised data collection, and critically reviewed the manuscript for important intellectual content. All authors approved the final manuscript as submitted and agree to be accountable for all aspects of the work.

Funding AP-G and IL-C are both research fellows granted by the Instituto de Investigación Sanitaria La Fe; AP-L acknowledges a Rio Hortega predoctoral grant (CM18/00165) from the Instituto de Salud Carlos III (Ministry of Science and Innovation, Spain); MV and AS-G pertain to the RETICS funded by the PN 2018-2021 (Spain), ISCIIISub-Directorate General for Research Assessment and Promotion and the European Regional Development Fund (FEDER), reference RD16/ 0022 .

\section{Compliance with ethical standards}

Conflict of interest The authors declare no competing interests.

Publisher's note Springer Nature remains neutral with regard to jurisdictional claims in published maps and institutional affiliations.

\section{References}

1. Garland JS. Strategies to prevent ventilator-associated pneumonia in neonates. Clin Perinatol. 2010;37:629-43.

2. Geslain G, Guellec I, Guedj R, Guilbert J, Jean S, Valentin C, et al. Incidence and risk factors of ventilator-associated pneumonia in neonatal intensive care unit: a first French study. Minerva Anestesiol. 2018;84:829-35.

3. CDC. Pneumonia (Ventilator-associated [VAP] and nonventilator-associated Pneumonia [PNEU]) Event. 2021.

4. Cernada M, Brugada M, Golombek S, Vento M. Ventilatorassociated pneumonia in neonatal patients: an update. Neonatology. 2014;105:98-107.

5. Cernada M, Aguar M, Brugada M, Gutiérrez A, López JL, Castell $\mathrm{M}$, et al. Ventilator-associated pneumonia in newborn infants diagnosed with an invasive bronchoalveolar lavage technique: a 
prospective observational study. Pediatr Crit Care Med. 2013;14:55-61.

6. Iosifidis E, Pitsava G, Roilides E. Ventilator-associated pneumonia in neonates and children: a systematic analysis of diagnostic methods and prevention. Future Microbiol. 2018;13:1431-46.

7. Dell'Orto V, Raschetti R, Centorrino R, Montane A, Tissieres P, Yousef $\mathrm{N}$, et al. Short- and long-term respiratory outcomes in neonates with ventilator-associated pneumonia. Pediatr Pulmonol. 2019;54:1982-8.

8. Sosa-Hernández O, Matías-Téllez B, Estrada-Hernández A, Cureño-Díaz MA, Bello-López JM. Incidence and costs of ventilator-associated pneumonia in the adult intensive care unit of a tertiary referral hospital in Mexico. Am J Infect Control. 2019;47:e21-5.

9. Gokce IK, Kutman HGK, Uras N, Canpolat FE, Dursun Y, Oguz SS. Successful implementation of a bundle strategy to prevent ventilator-associated pneumonia in a neonatal intensive care unit. J Tropical Pediatrics. 2018;64:183-8.

10. Weber CD. Applying adult ventilator-associated pneumonia bundle evidence to the ventilated neonate. Adv Neonatal Care. 2016;16:178-90.

11. Azab SFA, Sherbiny HS, Saleh SH, Elsaeed WF, Elshafiey MM, Siam AG, et al. Reducing ventilator-associated pneumonia in neonatal intensive care unit using "VAP prevention Bundle": a cohort study. BMC Infect Dis. 2015;15:4-10.

12. Chapman AK, Aucott SW, Milstone AM, Esteban E, Ferrer R, Urrea $\mathrm{M}$, et al. Ventilator-associated pneumonia in neonates and children: a systematic analysis of diagnostic methods and prevention. Future Microbiol. 2012;13:1431-46.

13. World Health Organization. WHO guidelines on hand hygiene in health care first global patient safety challenge clean care is safer care. World Health Organization; Geneva; 2017.

14. AACN. Ventilator-associated pneumonia precautions for children: what is the evidence? Adv Crit Care. 2019;30:68-71.

15. VD. R. International Nosocomial Infection Control Consortium report, data summary of 50 countries for 2010-2015: deviceassociated module. Am J Infect Control. 2016;44:1495-504.

16. Esteban E, Ferrer R, Urrea M, Suarez D, Rozas L, Balaguer M, et al. The impact of a quality improvement intervention to reduce nosocomial infections in a PICU. Pediatr Crit Care Med. 2013;14:525-32.
17. Rosenthal VD, Álvarez-Moreno C, Villamil-Gómez W, Singh S, Ramachandran B, Navoa-Ng JA, et al. Effectiveness of a multidimensional approach to reduce ventilator- associated pneumonia in pediatric intensive care units of 5 developing countries: International Nosocomial Infection Control Consortium findings. Am J Infect Control. 2012;40:497-501.

18. Azab SFA, Sherbiny HS, Saleh SH, Elsaeed WF, Elshafiey MM, Siam AG, et al. Reduction of nosocomial infections and mortality attributable to nosocomial infections in pediatric intensive care units in Lithuania. Medicina. 2015;45:4-10.

19. Conway Morris A, Kefala K, Simpson AJ, Wilkinson TS, Everingham K, Kerslake D, et al. Evaluation of the effect of diagnostic methodology on the reported incidence of ventilator-associated pneumonia. Thorax. 2009;64:516-22.

20. Ergenekon E, Çataltepe S. Ventilator-associated pneumonia in the NICU: time to boost diagnostics? Pediatr Res. 2020;87:1143-44.

21. Parker LA, Weaver M, Murgas Torrazza RJ, Shuster J, Li N, Krueger C, et al. Effect of gastric residual evaluation on enteral intake in extremely preterm infants: a randomized clinical trial. JAMA Pediatr. 2019;173:534-43.

22. Lin Y, Sun Z, Wang H, Liu M. The effects of gastrointestinal function on the incidence of ventilator-associated pneumonia in critically ill patients. Open Med (Wars). 2018:556-61.

23. Düzkaya DS, Yildiz S Effect of two different feeding methods on preventing ventilator associated pneumonia in the paediatric intensive care unit (PICU): a randomised controlled study. Aust Crit Care. 2015:1-7.

24. Manzoni P, de Luca D, Stronati M, Jacqz-Aigrain E, Ruffinazzi G, Luparia M, et al. Prevention of nosocomial infections in neonatal intensive care units. Am J Perinatol. 2013;30:81-8.

25. Brierley J, Highe L, Hines S, Dixon G. Reducing VAP by instituting a care bundle using improvement methodology in a UK paediatric intensive care unit. Eur J Pediatrics. 2012;171:323-30.

26. Pepin BJ, Lesslie D, Berg W, Spaulding AB, Pokora T, HarrisHaman PA, et al. ZAP-VAP: a quality improvement initiative to decrease ventilator-associated pneumonia in the neonatal intensive care unit, 2012-2016. Adv Neonatal Care. 2019;19:253-61.

27. Al-Alaiyan S, Lee PL, Lee W, Chen HL, Garland JS, Conway Morris A, et al. Ventilator associated pneumonia in a neonatal intensive care unit. Thorax. 2017;15:16-21. 